\title{
Remark on "On Error-free Filtering of Finite-State Singular Processes under Dependent Distortions" Published in Probl. Peredachi Inf., 2007, no. 4
}

\author{
V. V. Prelov and E. C. van der Meulen
}

DOI: $10.1134 /$ S0032946009020100

The authors thank S.A. Pirogov for finding an error in the proof of the main theorem of our paper "On Error-free Filtering of Finite-State Singular Processes under Dependent Distortions" (Probl. Peredachi Inf., 2007, vol. 43, no. 4, pp. 271-279). The statement of the theorem remains valid (even without additional assumption on finiteness of the number of values of a completely singular process $X)$ if $Y$ is an output process of a stationary memoryless channels whose input signal is $X$. However, in the general case, where $Y$ is absolutely regular with respect to $X$, the question of validity of the theorem statement remains open.

February 25, 2009.

V.V. Prelov, E.C. van der Meulen 